\title{
Safety of transgenic corn
}

SIR - We feel that a few clarifications are needed on the main topic of a recent leading article and the News story by Meredith Wadman (Nature 383, 559 \& 564; 1996).

(1) Ciba's transgenic corn is first and foremost an insect-control tool provided to farmers so they can protect their crops against one of the most devastating corn pests, the European corn-borer. Both articles emphasized the herbicide-resistance trait as the main feature of Ciba's transgenic corn (maize).

(2) Britain's Advisory Committee on Novel Foods and Processes was concerned only about the remote possibility of transfer of the nonfunctional $\beta$-lactamase DNA sequence from Ciba transgenic corn to gut microbes of livestock to which it is fed. Some of your comments extended this concern to the possibility of transfer to humans, thereby presenting an inaccurate view of this issue.

(3) The risk of transfer of $\beta$-lactamase DNA from plant material to gut microflora of livestock is vanishingly small, and is far outweighed by the economic, environmental and feed safety benefits of this product. The only country that has so far formally raised the transfer of ampicillin resistance as an issue is the United Kingdom. Ciba's Bt corn has been reviewed and fully approved by the US, Canadian and Japanese governments. In addition, the French authorities who acted as rapporteur for our dossier in Europe also recommended full commercial approval. Genuine scientific debate is taking place, and outside the United Kingdom there is a scientific consensus emerging on the topic which was not echoed in your leading article. Several prominent independent scientists have expressed their views on the subject. Furthermore, the Foundation for Nutritional Advancement and Tufts University as well as a joint FAO/WHO Expert Consultation on Biotechnology and Food Safety concluded that the issue of ampicillinresistance transfer from a plant to a microbe constituted a near-zero risk of creating ampicillin-resistance complications. Antibiotic-resistant microbes are much more likely to occur by DNA transfer from one bacterium to another in nature.

(4) The "aggressiveness" that you correctly ascribed to Ciba reflects our strong affirmation of the scientific consensus on the safety of our Bt corn. Contrary to the impression left by your leading article that Ciba has remained insensitive to concerns raised by advisory and regulatory committees, Ciba's scientific and regulatory staff have worked cooperatively at every opportunity with the appropriate committees in matters concerning the benefits and safety of genetically modified crops worldwide. In this regard, Ciba's approvals for production and commercialization of its transgenic corn in the countries mentioned have been obtained through a cooperative effort using sound scientific data and rationale to address the various issues and concerns.

Juan J. Estruch

Mary-Dell Chilton

Richard Lotstein*

Ciba Biotechnology,

3054 Cornwallis Road,

Research Triangle Park,

North Carolina 27709-2257, USA

Wallace Beversdorf

Ciba Ltd, Seed Division,

Basel, Switzerland

*To whom correspondence should be addressed.

\section{Live universes}

SIR - In their recent Commentary article (Nature 384, 107; 1996), John Maynard Smith and Eors Szathmary consider the idea (proposed by L. Smolin and J. A. Wheeler) that universes are 'alive' in the sense that they can pass on hereditary information, show variability from one generation to the next, and are subject to selective pressures. It seems ironic that evolutionary biologists should take such an idea seriously enough to comment (even sceptically) on it, while at the same time treating with derision the much less radical proposals that ecosystems or planets (in particular, Earth) may also be alive.

The possibility that life forms exist at levels above that of individual organisms is generally denied because these higher-order systems fail one or more of the criteria established for the recognition of life. But this seems tautological; there is no reason to expect that super-organisms would meet criteria based on observations of individual organisms. Isn't it time to consider the possibility that the boundary between life and non-life may be diffuse, non-stationary over time, and dependent upon scale?

\section{G. Bjornerud}

Geology Department,

Lawrence University,

Appleton, Wisconsin 54912, USA

e-mail: bjornerm@lawrence.edu

SIR - Maynard Smith and Szathmary say that the simplest interpretation of the strong anthropic principle is that the Universe was created purposefully. They make the intriguing claim that "this interpretation lies outside science" and turn their attention to more complex and (one presumes) more 'scientific' interpretations.

What do they mean by stating that a hypothesis is "outside science"? They offer no justification for this bald statement and make no attempt to persuade the reader that the other hypotheses under considera- tion are in any sense better or more meaningful. Simply to dismiss a concept as being beyond analysis seems a rather crude form of intellectual censorship. Those who seek to address the possibility of God's existence in a rational way are thereby disenfranchised.

If the Universe has been purpose-built, one might expect there to be evidence of this. At the very least we should address the data without excluding this possibility. We may or may not see God's signature, but we owe it to ourselves to try to keep our eyes open.

Graeme Suthers

South Australian Clinical

Genetics Service,

Centre for Medical Genetics, Women's \& Children's Hospital, North Adelaide,

South Australia 5006

e-mail: suthersg@wch.sa.gov.au

\section{Out of context}

SIR - In the space of one paragraph, George Magyar (Nature 384, 509; 1996) accuses me of trying to discredit science and three of its more notable contemporary practitioners: Steven Weinberg, Herbert Simon and Noam Chomsky. He makes this accusation in the context of complaining about the unscholarly behaviour of sociologists of science. Unfortunately, his references to my work in Social Studies of Science are either completely spurious or twisted out of context.

It is true that I have had occasion to criticize Weinberg and Simon in reviews of their work (as part of an open exchange with them). I suggested, in the case of Weinberg, that to identify science exclusively with what élite scientists do is comparable to "megalomania" (Soc. Studies Sci. 24, 154; 1994). In the case of Simon, when I said he had a "muddled grasp of the metatheoretical issues surrounding his work" ( 21, 149; 1991), I was simply making a point that philosophers have raised before, namely that Simon equivocates over whether his computer simulations are meant to describe or to prescribe the processes of rational thought. As for Chomsky, my reference to him as a "linguistic upstart" $(19,629 ; 1989)$ was not a judgement about the quality of his work but a description of how behaviourists initially viewed him when he launched his famous attack on B. F. Skinner.

I am content to let readers draw their own conclusions about my claims in the context of the original discussions.

\section{Steve Fuller}

Department of Sociology

\& Social Policy,

University of Durham,

Durham DH1 3JT, UK

e-mail: steve.fuller@durham.ac.uk 